\title{
Importance des interventions primaires P1
}

Peter Matter, Susanne Imbach, Mark Brouwer, Beat Hugentobler, Ursula Loeliger

Etude soutenue par une contribution financière de la part du fonds de recherche de la Suva.

Correspondance:

Prof. Dr Peter Matter

Ortstrasse 6

CH-7270 Davos Platz

Tél. 0814133286

peter_matter@bluewin.ch

\section{Introduction}

Le fait que les travaux sur la définition de normes de qualité pour le secourisme ne puissent, en Suisse, se fonder sur des études scientifiques aptes à démontrer l'efficacité des mesures ordonnées et prises dans le contexte d'une intervention de sauvetage ne va pas sans poser des problèmes. Des informations fiables sur les coûts engendrés par la mise à disposition de prestations dans le domaine du sauvetage font également défaut.

L'Interassociation de sauvetage estime qu'il est grand temps de combler ce manque d'informations sur l'efficacité des mesures prises dans le domaine du sauvetage. C'est bien pour cette raison qu'elle a mené une étude sur l'ensemble des opérations de sauvetage, conduisant de l'alarme donné à la centrale d'appels sanitaires urgents à l'intervention des services de sauvetage jusqu'à l'hospitalisation du patient. Les centrales d'appels sanitaires urgents 144 ont, lors de chaque appel, la lourde responsabilité de devoir trancher s'il s'agit d'une «urgence avec probabilité d'atteinte des fonctions vitales» nécessitant une intervention P1 avec signaux prioritaires et recours à un médecin d'urgence. Pareille opération entraîne des coûts élevés.

\section{Objectifs}

Le but de l'étude a été de mettre en évidence les avantages et les inconvénients du système actuel, de définir les corrections nécessaires et, par la suite, au besoin, de procéder à un remaniement des normes de qualité.

\section{Termes de l'étude}

L'étude avait pour objectif de trouver une réponse aux questions suivantes:

- justification des interventions P1;

- dotation en personnels et leur qualification;

- comparaison NACA et GCS entre le site de l'incident et l'hôpital;

- délais de réponse et délais d'intervention en rapport avec la distance parcourue;

- comparaison des résultats entre les CASU impliquées dans l'étude et l'ensemble de celles-ci.

\section{Aspects déterminants pour l'avenir}

- Mise au point d'une méthode spécifique pour la saisie des données par les différents partenaires impliqués dans la chaîne de sauvetage (centrales d'appels sanitaires urgents, services de sauvetage, hôpitaux) et sa transposition scientifique.

- Tous les participants utilisent la même terminologie uniforme.

- Mise sur pied d'un système de communication suffisamment transparent pour les centrales d'appels sanitaires urgents, les services de sauvetage et les hôpitaux.

\section{La mise sur rail de l'étude}

Onze CASU ont pris la décision de participer volontairement à l'étude. Celles-ci y ont associé les services de sauvetage ainsi que les hôpitaux sis à l'intérieur de leur rayon d'action. L'étude englobe toutes les interventions primaires $\mathrm{P} 1$ ordonnées par les CASU entre le $1^{\text {er }}$ et le $5^{\mathrm{e}}$ jour de chaque mois. Après une phase d'essai l'étude prit son départ le $1^{\text {er }}$ décembre 2004, portant de décembre 2004 à mars 2005 sur les cinq premiers jours de chaque mois. Les données à saisir tablaient sur la terminologie et sur le schéma de saisie chronologique des données de l'IAS. Les données à saisir ont été anonymisées dès le début, en vue d'en assurer la protection telle que requise. L'étude a été menée avec l'autorisation préliminaire de la «Commission d'experts du secret professionnel en matière de recherche médicale».

\section{Résultats}

633 protocoles complets des CASU et des services de sauvetage étaient entrés au terme de l'étude. Ils contiennent au total 363 protocoles rédigés par les hôpitaux de destination.

Dans $68 \%$ des cas, l'intervention urgente a été demandée par des profanes (fig. 1). Le dispositif des interventions a été presque exclusivement conçu par des régulateurs qualifiés. C'est le «Advanced Medical Priority Dispatch System» (AMPDS) qui a été appliqué dans un tiers des cas. Les délais de traitement des appels d'urgence n’ont, dans $77 \%$ des cas, pas dépassé les 2 minutes exigées par les directives (fig. 2).

Dans $88 \%$ des cas, les délais de réponse se situent à l'intérieur de la plage des 15 minutes exigées, l'étude n'ayant toutefois pas permis de faire une distinction entre agglomération et périphérie (fig. 3). Les délais moyens et les intervalles (fig. 4) correspondent sur toute la ligne aux délais de traitement d'appels d'urgence et d'inter- 
vention souhaités. Le tableau révèle toutefois également des maximums qui devront faire l'objet d'un examen approfondi fondé sur l'analyse des différents protocoles. En ce qui concerne la saisie chronologique des données en relation avec les kilomètres parcourus, (fig. 5), on constatera avec étonnement que dans 188 cas, le trajet jusqu'au site de l'incident dépasse les $10 \mathrm{~km}$ et que dans 197 cas le transport du site de l'intervention vers la destination du patient en détresse excède également les $10 \mathrm{~km}$. Il conviendra de mener à ce sujet une analyse plus fouillée en vue de déterminer le meilleur emplacement possible des ambulances et d'optimiser le choix des hôpitaux de destination. $7 \%$ des protocoles font, en relation avec le temps de séjour sur le site de l'incident, état de conditions difficiles de dégagement du patient.

$37 \%$ des interventions se sont faites avec participation d'un médecin qui, dans $26 \%$ des cas, avait été mobilisé par la CASU (fig. 6). Dans 65\% des interventions avec médecin, celui-ci a rejoint le site avec l'ambulance, dans $27 \%$ par le truchement du système dit «rendez-vous» et dans $8 \%$ des cas il a été mobilisé ultérieurement par l'équipe de sauvetage. Dans 60\% des interventions les médecins étaient anesthésistes, un tiers environ était titulaire d'un certificat d'aptitude de «médecin d'urgence». Les Directives de l'IAS concernant la reconnaissance des services de sauvetage exigent pour les interventions P 1 la présence d'un ambulancier catégorie $\mathrm{A}$ et, en plus, d'une personne avec formation d'un an au minimum. Cette règle minimale était remplie dans $94 \%$ des interventions avec médecin, et dans $93 \%$ des interventions sans présence d'un médecin (fig. 7).

La détermination routinière de deux scores se fit dans un premier temps sur le site de l'incident, dans un deuxième temps lors de l'entrée du patient à l'hôpital de destination (fig. 8). L'indice NACA était dans $63 \%$ des interventions inférieur à 4 sur le site de l'incident, sans présenter de variations notables jusqu'à l'arrivée à l'hôpital. L'échelle GCS, mesurée sur le site de l'incident, ne présenta pour $64 \%$ des patients pas de déviation par rapport à la norme, le score parvenant à légèrement s'améliorer jusqu'au moment de l'hospitalisation.

$\mathrm{Au}$ terme de l'appréciation donnée par le médecin au moment de l'entrée du patient à l'hôpital de destination, l'intervention P 1 avec signaux prioritaires se révéla être justifiée dans $75 \%$ des cas alors que, dans $25 \%$ des cas, elle était ultérieurement jugée problématique.

L'examen entrepris sur le site (fig. 9) permit de diagnostiquer dans $70 \%$ des cas une maladie, pour les autres $30 \%$, il s'agissait d'accidents. Les principales maladies étaient pour $14 \%$ des accidents coronaires, pour $13 \%$ des altérations de la conscience. Les accidents de la circulation se chiffrèrent à $8 \%$, les autres accidents à $21 \%$, les brûlures ayant une part de $1 \%$. Pour $20 \%$ des patients, les fonctions vitales étaient toujours menacées au moment de leur hospitalisation.

Les deux facteurs sur la base desquels il fut tenté de mesurer la qualité des résultats étaient les chances de réhabilitation ainsi que le risque d'une atteinte durable à la santé (fig. 10). 78\% des cas pouvaient s'attendre à une réhabilitation intégrale; pour $84 \%$ des patients, l'issue du traitement ne devait pas leur laisser d'atteinte durable à la santé. Il n'a pas été possible de déterminer avec précision le nombre de décès, étant donné qu'aux victimes mortes sur place, s'ajoutaient les sujets transportés sous réanimation ou pour constat de décès dans un hôpital de destination.

\section{Discussion}

L'étude et son dépouillement ont été planifiés avec soin; les données à caractère prospectif ont rendu possible des analyses qui pourront être instrumentalisées par la suite. Les 633 protocoles complets rédigés par les CASU et les services de sauvetage ainsi que les 363 enquêtes menées dans les hôpitaux dépassent les objectifs numériques fixés au préalable. Pendant que l'étude était en cours, les résultats obtenus ont été soumis plusieurs fois à examen, ce qui a permis de constater une très grande stabilité des pourcentages enregistrés. Ils peuvent donc être considérés comme représentatifs.

Le nombre élevé d'appels de secours faits par des profanes démontre à l'évidence à quel point il est difficile pour le régulateur de la CASU de décider de l'opportunité d'une intervention à caractère prioritaire. Il importe donc de dûment enseigner le déclenchement correct de l'alarme. Les régulateurs devraient pouvoir prendre leurs décisions sur la base d'une check-list largement uniformisée. Les démarches pourraient ainsi être optimisées, puisqu'on disposerait alors d'une évaluation objective et de possibilités de comparaison. Les délais de traitement des appels d'urgence, les délais de départ, la moyenne des délais et les intervalles mesurés mettent largement en évidence la rapidité et l'efficacité des interventions urgentes. Les délais de traitement des appels supérieurs à 3 minutes (10\%) et les délais de départ des services de sauvetage de plus de 5 minutes (7\%), ainsi que les délais de réponse dépassant les 71 minutes et les délais d'intervention de plus de 174 minutes devront nécessairement, de cas en cas, faire l'objet d'une analyse 
Documents de travail

- Directives de l'IAS concernant la reconnaissance des services de sauvetage du 3 février 2000 avec amendements du 11 mars 2004.

- Directives de l'IAS concernant la reconnaissance des centrales d'appels sanitaires urgents du 6 juin 2002.

- Terminologie dans le domaine du sauvetage IAS/SMEDREC du 24 avril 2003.

- Fiches d'intervention IAS du 2 décembre 1999, état janvier 2002 .

- Autorisation de la part de la «Commission d'experts du secret professionnel en matière de recherche médicale». 17 juin 2004 approfondie, et ce dans le but de les corriger. Cet examen pourrait éventuellement, en vue de réduire les distances d'intervention, aboutir à un changement du lieu de stationnement des ambulances et/ou à un autre choix des hôpitaux de destination.

La question de l'inclusion d'un médecin dans l'opération de secours n'a pas encore été tranchée et devra faire l'objet d'un examen approfondi. Dans toute la mesure du possible, c'est la CASU qui devrait faire appel au médecin. L'étude a également permis de constater que la doctrine des interventions P1 était objet d'interprétations très divergentes. La définition de l'intervention P1 ne devrait pas être confinée au seul facteur de la «probabilité ou de la présence d'une atteinte des fonctions vitales». Mieux différenciée, celleci rendrait possible une participation plus sélective du médecin à l'opération de secours. Il faudra attendre l'issue du débat sur les qualifications professionnelles, actuellement en cours au sein du Forum formation professionnelle, avant de pouvoir édicter des directives claires et précises sur la composition des équipes de secours. Les directives présentes sur la composition des équipages sont respectées dans plus de $90 \%$ des cas.

Seul pour un tiers des alertes déclenchées, les interventions P1 se faisaient en présence d'un indice NACA 4 ou supérieur. Ce fait met en évidence la nécessité de revoir les définitions données par les CASU aux interventions P1. Un accident de la circulation, pour ne citer qu'un exemple, nécessite presque toujours une inter- vention P1, sans que celle-ci ne doive nécessairement se fonder sur un indice NACA élevé. Les résultats enregistrés sur l'échelle GCS doivent être interprétés en analogie. L'espérance d'une réhabilitation intégrale dans $78 \%$ des cas ainsi qu'une issue des traitements sans atteinte durable à la santé dans $84 \%$ des cas sont des résultats certes satisfaisants. Pour que les futures études soient encore plus concluantes, il conviendra de les faire reposer sur un nombre plus élevé d'indicateurs de résultat. Les hôpitaux devraient, en outre, se résoudre à surmonter leur réticence face à la mise à disposition d'informations. Seul de cette manière sera-t-il possible de procéder à une évaluation plus nuancée des résultats ainsi qu'à une optimisation des interventions.

\section{Conclusions}

L'étude de l'IAS sur les interventions P1 permet de conclure aux démarches suivantes:

- formation poussé du déclenchement correct et schématique de l'alarme;

- uniformisation des dispositifs d'intervention dans les CASU;

- définition plus nuancée de l'intervention prioritaire $\mathrm{P} 1$;

- différenciation de la liste des indications en faveur d'une intervention du médecin;

- mobilisation du médecin par la CASU;

- reconsidération de l'équipage des ambulances;

- évaluation commune par les CASU, les services de sauvetage et les hôpitaux de la qualité des résultats des opérations précliniques.

Figure 1

Première alerte centrale d'appels d'urgence.

\section{Premiere alerte}

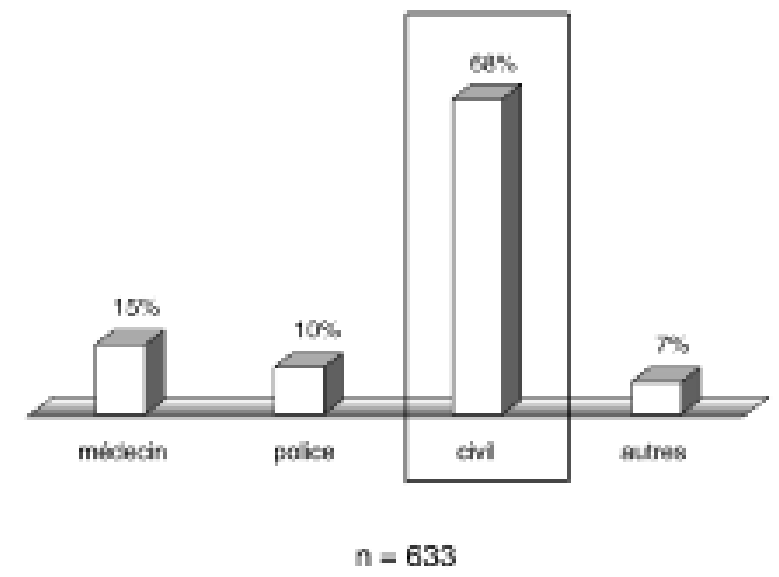


Figure 2

Délais de traitement d'appel d'urgence (CASU), délais de départ (service de sauvetage).

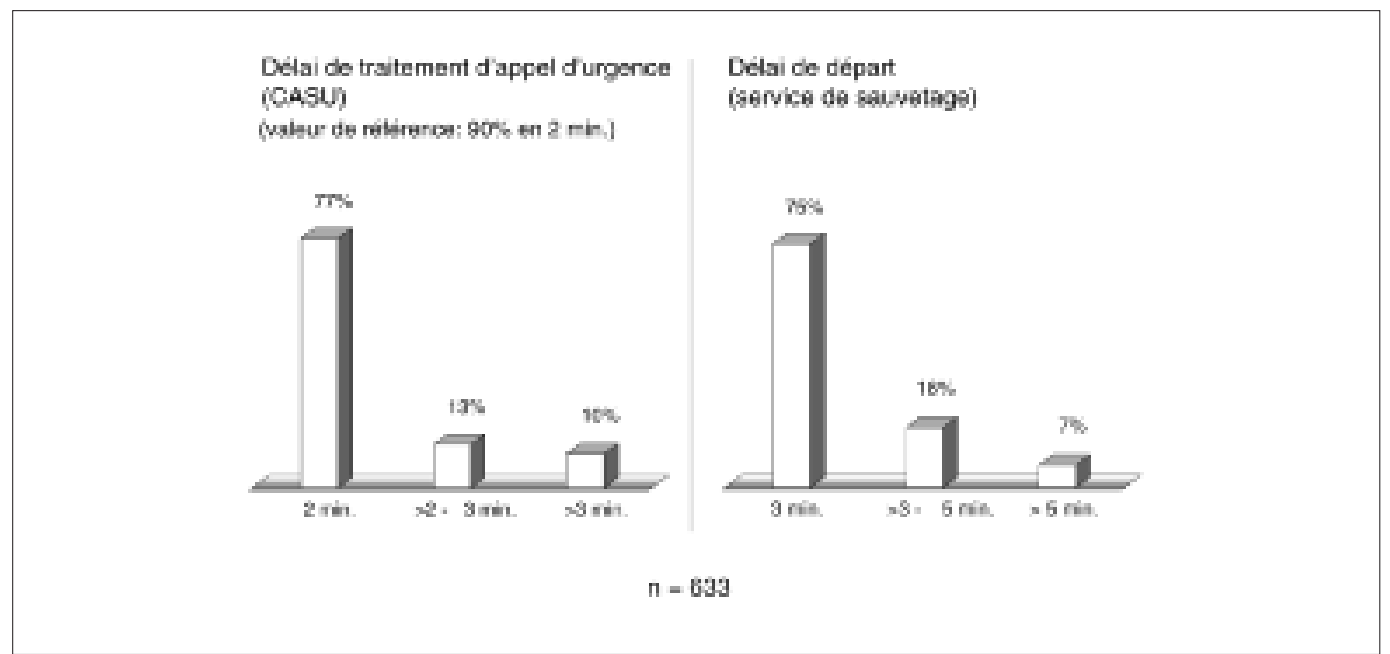

Figure 3

Délais de réponse et temps sur site.

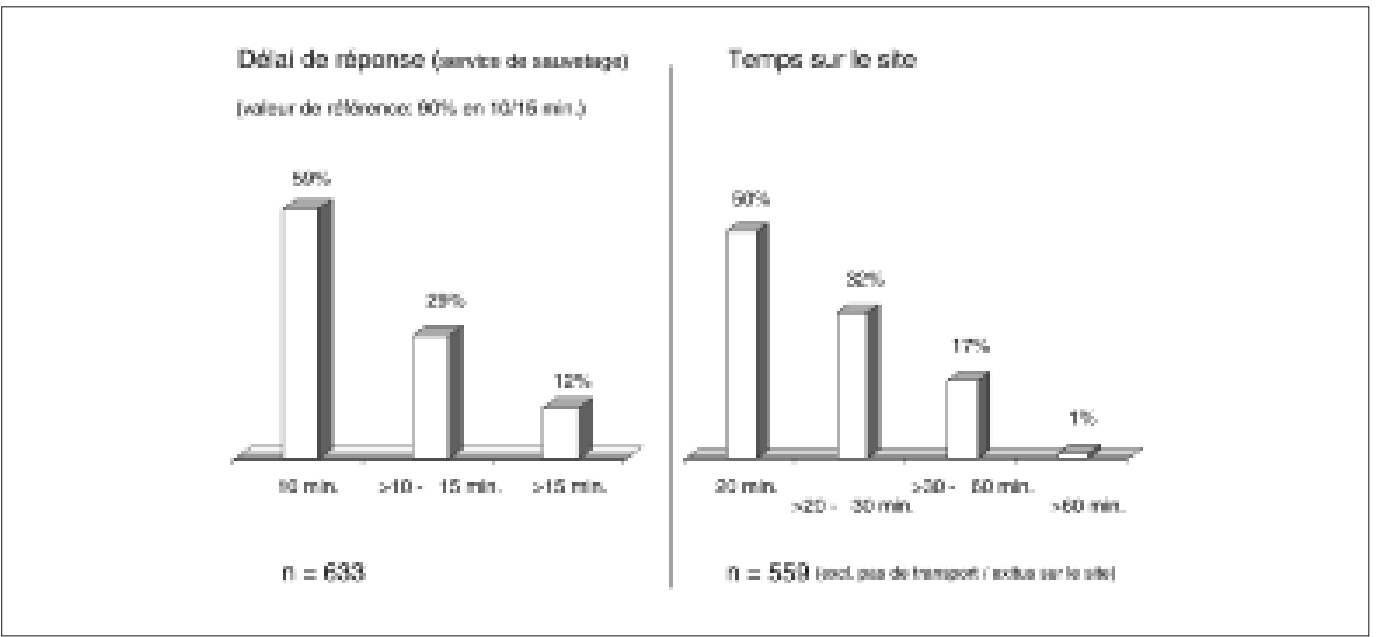

Figure 4

Moyenne des délais et des intervalles, maximums et minimums inclus.

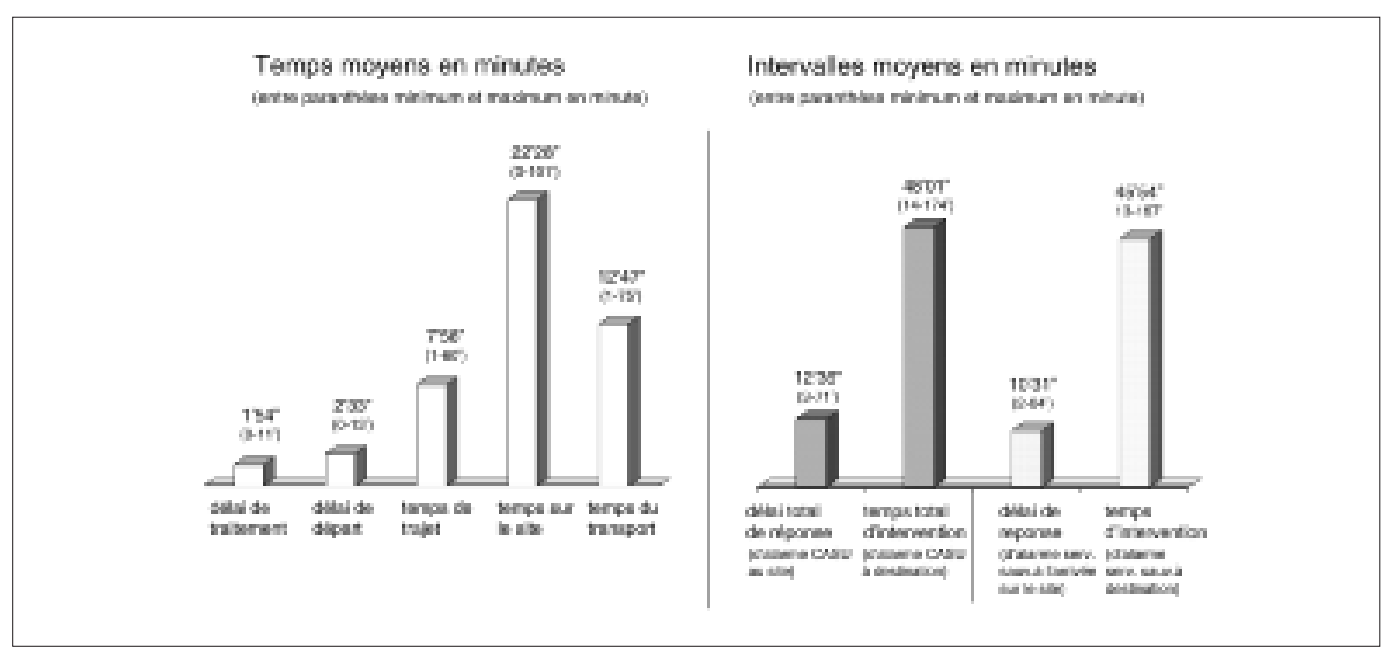


Figure 5

Délais de réponse moyens par rapport à la distance.

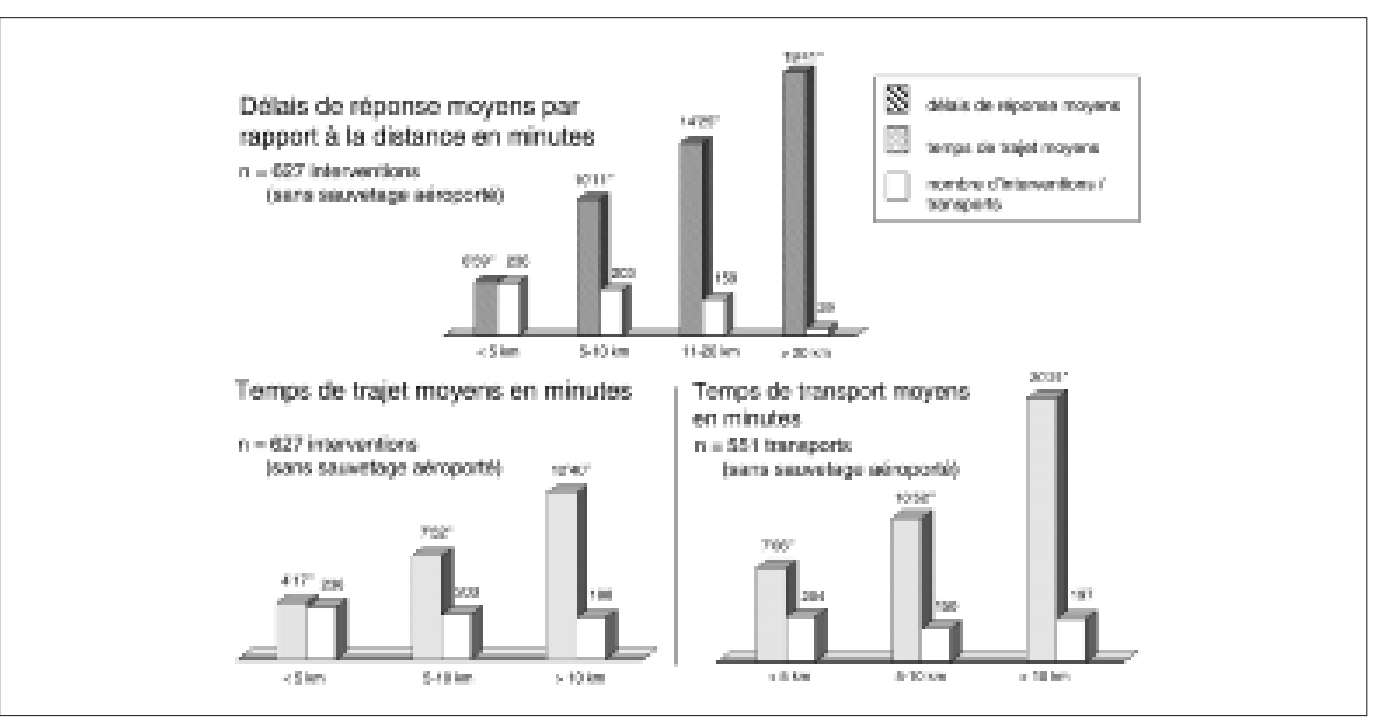

Figure 6

Intervention avec médecin

Imervernions arec mbdacin Charactéristique de fintervention

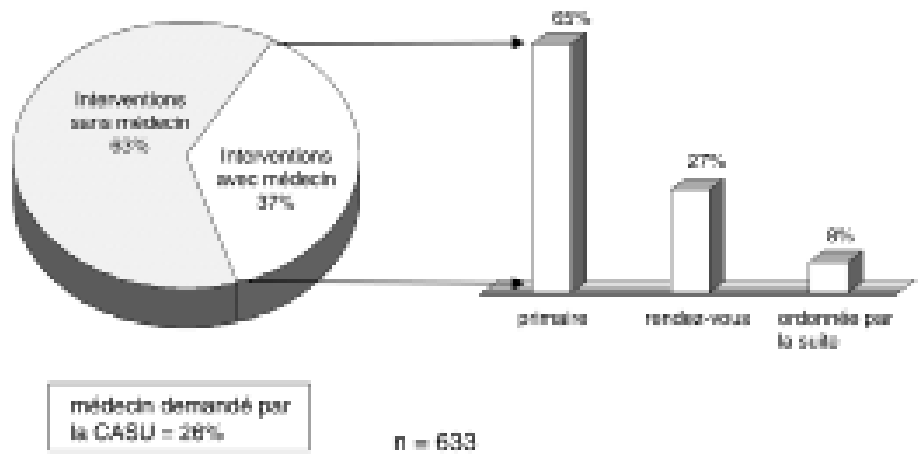

Figure 7

Attribution du personnel et qualification.

\section{Attribution du personnel \\ Qusitcation du personel}

au min. 1 personne de la cat. A dans l'bquipe

avec 1 personne de la cat. A, B ou C (solon les valeurs de rberance)

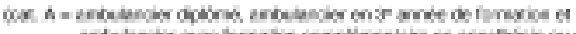

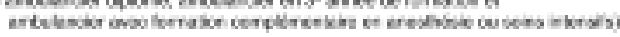

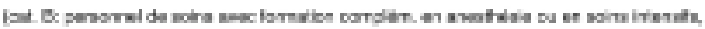

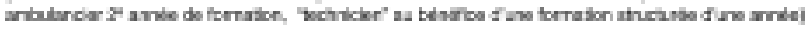

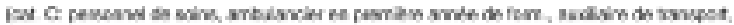

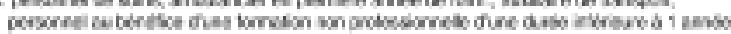

\section{6 nterventions avec mbdecin sur le ste:}

min. 1x aut. $A+1 \times A B C=94 \%$

\section{7 interventions sans} modecin sur le she:

min. $1 x$ cat. $A+1 \times$ Ma/C $=98 \%$

$n=633$ 
Figure 8

Indices NACA et GCS sur le site.

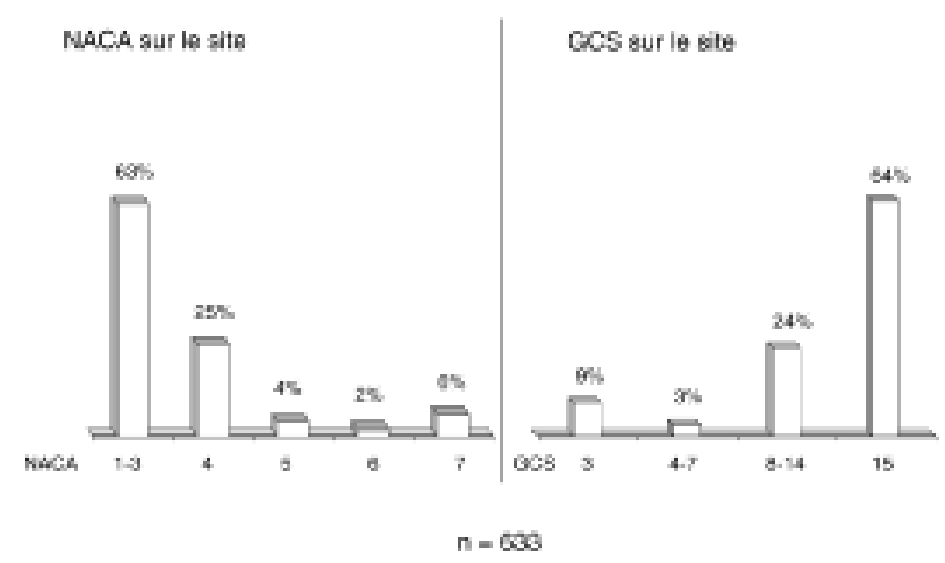

Figure 9

Etat du patient sur le site.

Etat du patient sur le lieu de févínement

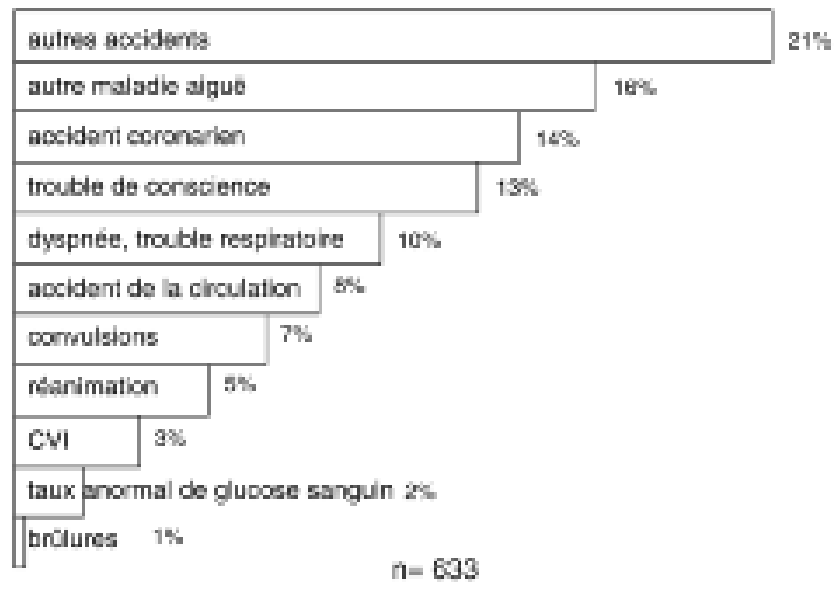

Figure 10

Rétablissement et résultats de traitement.

Riglablisement

